\title{
Reacting to the Reaction of the Reaction to the Reaction
}

\author{
Reaction Videos und invektive Blickzirkulationen
}

Johanna Heyne

\begin{abstract}
Seit einigen Jahren ist es aufder Plattform YouTube zum Trend geworden, dass sich User:innen dabei filmen, wie sie sich wiederum Filme im Internet anschauen. Die sogenannten Reaction Videos generieren vor allem dann Aufmerksamkeit und Reichweite, wenn die Gefilmten aufbesonders drastische Inhalte reagieren. Die Vermittlung der Bilder durch die Reaktion der Gefilmten schafft eine doppelte Beobachtungsebene, die im Kontext der sozialen Medien in normierende Prüfungen und Wertungen eingebunden ist. Der Beitrag widmet sich diesem Phänomen und entwickelt anhand der Überlegungen zum Blick, zur Scham und zum Ekel von Sartre, Lacan, Kristeva und Butler eine Heuristik invektiver Blicke erster und zweiter Ordnung.
\end{abstract}

\section{Einleitung}

Am 15. November 2017 veröffentlichte das Auktionshaus Christie's auf der Videoplattform YouTube einen Trailer zur Versteigerung des Leonardo da Vinci zugeschriebenen Salvator Mundi: Vor dunklem Hintergrund betrachten Menschen etwas, das sich außerhalb des filmischen Rahmens befindet. Ein Licht scheint von dem geheimnisvollen Objekt auszugehen und erhellt die Gesichter der Personen. Etwas bannt ihren Blick. Einige der Schauenden sehen ehrfürchtig aus, einige lächeln, sind zu Tränen gerührt. Dazu erklingt die getragene Musik eines Streichorchesters (vgl. Christie's 2017) (Abb. 1-3).

Was hier als Marketinginstrument eines großen Auktionshauses genutzt wurde, ist ursprünglich auf YouTube als sogenanntes Reaction Video populär geworden. Dabei ist die gezeigte Konstellation immer gleich: Die Reaktionen sind über die Webcam oder externe Kamera so gefilmt, dass sie den Bildschirm und das Video, auf das reagiert wird, nicht zeigen, sondern nur die Gesichter der Schauenden sichtbar machen. So sind die Sichtbarkeitsverhältnisse der Reaction Videos eine logische Folge ihrer technischen Produktionsbedingungen. 
$A b b .1$ und Abb. 2
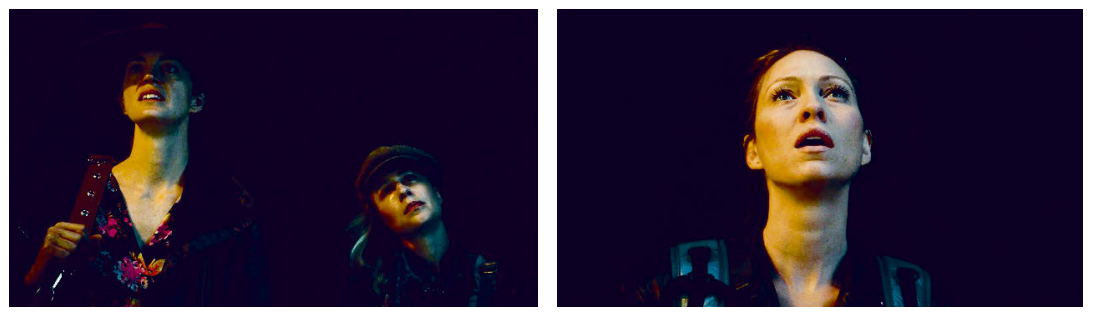

Nach dem Hype auf YouTube entwickelte sich das Reaction Video über die Plattform hinaus zu einem eigenständigen popkulturellen Phänomen. Es etablierte sich ein Genre von Blickkonstellationen mit besonderem affektiven Potenzial, das gerade deshalb zur zirkulierenden Ware wird.

Abb. 3

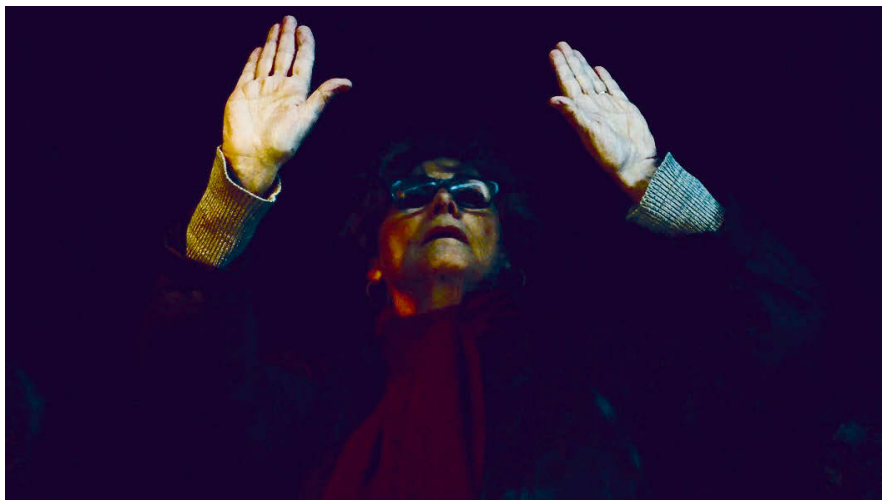

Reaction Videos auf YouTube sind unabgeschlossene Produkte, die in ein komplexes System von Bewertungs- und Reaktionsmöglichkeiten eingespannt sind, das seinerseits produktive Rezeptionshaltungen hervorruft. Die Analyse der zugrunde liegenden medialen, affektiven und intersubjektiven Prozesse sowohl in der Produktion als auch der Rezeption ist daher für das Verständnis dieser Videos entscheidend.

Vor allem die Rezeption von Reaction Videos wird durch das dortige Interface kanalisiert. Neben Social Signals wie Like- und Dislike-Buttons oder der Möglichkeit, Videos zu teilen, ist auf YouTube und anderen sozialen Netzwerken die Kommentarfunktion zentral. Die Aufmerksamkeitsökonomie der Plattformen, in der 
Popularität und Anerkennung maßgeblich Handeln und Selbstdarstellung bestimmen, sind in besonderem Maße auf drastische Inhalte angewiesen, um wiederum starke Reaktionen und Emotionen auszulösen. Zudem ermöglichen sie den User:innen eine moralische Positionierung mittels Selbstdarstellungen. Aus diesem Grund reagieren die Gefilmten mit Vorliebe auf Videos, die besonders extreme Inhalte wie z.B. Hardcore-Pornografie, heftige physische Gewalt oder sogar Mord zeigen.

Die Praxis, mit diesen drastischen Bildern unter den Bedingungen digitaler Infrastrukturen umzugehen, ist im folgenden Beitrag von besonderem Interesse. Ein wesentliches Merkmal dieser Infrastrukturen, so werde ich herausarbeiten, sind die spezifischen Produktions-, Rezeptions- und Kommunikationsbedingungen von Sozialen Medien, aus deren Logik heraus und für deren neuerliche Verwertung drastische Bilder überhaupt erst erzeugt werden. Reaction Videos zu derart extremen Inhalten und ihre Rezeption sind maßgeblich von diesen Bedingungen beeinflusst. Genauso können Reaction Videos nur im Spannungsfeld zwischen einer vorher nicht dagewesenen Verfügbarkeit drastischer Bilder und der gleichzeitigen gesellschaftlichen Tabuisierung des Gesehenen verstanden werden.

Die Rezeption dieser Bilder durch die Gefilmten, so zeigt die Analyse der exemplarischen Reaction Videos, ist sehr oft ambivalent. Obwohl die Personen vor der Kamera starke Abwehrreaktionen zeigen, besteht doch ein scheinbarer Drang zum Schauen. Das beweist die hohe Zahl an Suchergebnissen, die YouTube für dieses Genre vorschlägt. Hinter diesem Zwang zum Hinsehen-Müssen trotz innerer Widerstände, so meine These, verbirgt sich eine Tradition des Schauens auf das Grauen, die ihre Anfänge schon lange vor den digitalen Bildern hat. Damit verbunden sind Blickstrategien, die sich zwischen Blickangst und Schaulust bewegen. Hier manifestiert sich eine erste Ebene des invektiven Blickes (invective gaze), indem nämlich Szenen der Herabsetzung betrachtet werden.

Reaction Videos, die sich auf gewaltvolle oder sexuelle Inhalte beziehen, erfordern von den Gefilmten immer auch eine moralische Positionierung. Denn um eine hohe Reichweite zu erzeugen, sind sie von einer möglichst positiven Bewertung ihrer Rezipient:innen abhängig. Die Social Signals machen die Aufmerksamkeit für ein bestimmtes Video und seine Beliebtheit oder Unbeliebtheit sicht- und messbar. Als phatische Kommunikationsmittel (vgl. Schäfer/Mühleder 2020: 130-149) unterstreichen sie den Aufforderungscharakter Sozialer Medien, sich ohne den Versuch, konkrete Information zu übermitteln unmittelbar an einem digitalen Diskurs zu beteiligen und die eigene Reaktion permanent zu dokumentieren. Über diese phatische Verbindung hinaus sind YouTube-Videos immer schon in ein affektives Bewertungsnetz eingebunden und insbesondere Reaction Videos stehen sinnbildlich für ein Kommunikationsregime, das explizit zur Beteiligung einlädt.

Dieses Regime durchdringt nicht zuletzt auch die Körper der YouTuber:innen, die sich für ihre Reaction Videos selbst filmen. Ihre affektiven und emotiven Äuße- 
rungen stellen sie vor einem Publikum zur Schau und werden so einer bewertenden Netzöffentlichkeit ausgesetzt. Die phatischen Mittel der Videoplattform fordern dazu auf, eine Reaktion zu hinterlassen. Auf diese Weise wird der Körper und seine Affekte adressiert und wird - auch wenn er physisch nicht präsent ist - im Digitalen verletzbar.

Ein besonders beliebtes Kommunikationsmittel ist die Kommentarfunktion. Die Kommentare setzen sich teilweise mit der Rezeptionshaltung der Gefilmten auseinander und sanktionieren als unpassend empfundenes Verhalten. Damit bieten sie wichtige Anhaltspunkte für die Untersuchung der Affektrezeption der zweiten Beobachtungsebene. Diese zweite Beobachtungsebene setzt sich nicht mehr mit den drastischen Bildern auseinander, sondern bezieht sich auf die im Video gezeigte Reaktion. Betrachtet wird hier also das Betrachten selbst. Diese Ebene ist zugleich die zweite Ebene des invektiven Blicks: Wenn die Kommentare unter den einzelnen Reaction Videos die Reaktionen der Gefilmten kritisieren, sich über die YouTuber:innen mokieren oder ein regelrechter Shitstorm über die Gefilmten hineinbricht, dann sehen sich die Produzent:innen der Reaction Videos einer öffentlichen Herabsetzung ausgesetzt.

Sowohl für das Setting der ersten als auch der zweiten Ebene des invektiven Blickes ist konstitutiv, dass das Schauen vor Publikum stattfindet. Nicht nur die Reaction Videos, sondern auch ihre Kommentierungen werden wiederum von einem Publikum rezipiert. Die User:innen publizieren also immer im Wissen um eine bewertende Netzöffentlichkeit und jede Handlung ist durch dieses Wissen beeinflusst. Warum also wird das Selbstexperiment der Reaction Videos immer aufs Neue öffentlich wiederholt und welche Rolle spielt dabei das unsichtbare Publikum?

Schon im Umfeld von YouTube setzen sich Netzakteure produktiv mit den eigenen Handlungsspielräumen auseinander, wenn sie mit neuen Produktionen auf bestehende Reaction Videos Bezug nehmen und endlose Reaktionsschleifen entstehen. Zudem verlässt das Bildphänomen mit zunehmender Popularität immer öfter seinen ursprünglichen Funktionszusammenhang und findet Eingang in Fernsehen, Werbung und sogar Kunst.

Der Bildtypus des Selfies ist in der Kunstgeschichte und Medienwissenschaft schon vielseitig beleuchtet worden (vgl. dazu u.a. Otto/Plohr 2015: 26-30; Reichert 2015: 86-96; Ulrich 2019). Auch das Reaction Video ist eine Form des Selfies, allerdings handelt es sich bei Reaction Videos um bewegte Bilder, während Foto-Selfies eher als Momentaufnahmen wirken. Es drängt sich die Frage auf, wie das reagierende Gesicht von einem Internetphänomen unter privaten User:innen zur medialen Folie für Unterhaltungsbranche, Kunst, und Werbung geworden ist und welche Strategien zu seiner Kommodifizierung beitragen. 


\section{Der invektive Blick erster Ordnung}

Die Firma MFX Media veröffentlichte 2007 den Pornofilm Hungry Bitches. Der Film war für ein Fetischpublikum als ein Scat Porn gedreht worden, also als pornografischer Film, der die erotische Lust am Spiel mit Ausscheidungen ausstellt. Vor der Veröffentlichung des Films wurde ein Trailer als Ankündigung online gestellt. Bald erzeugte dieser Trailer zahlreiche Reaction Videos, bei denen sich User:innen dabei filmen, wie sie sich den kurzen Clip anschauen. Die Reaktionen sind über die Webcam so gefilmt, dass der Bildschirm und der Trailer selbst nicht gezeigt werden, sondern nur die Gesichter der Schauenden sichtbar sind und so ein Reflex des Gesehenen über Mimik und Gestik vermittelt wird. Die Reaction Videos wurden für die Veröffentlichung auf YouTube produziert. Auf diese Weise fand der Trailer aus der Fetischszene des Scat Porns, der nun als 2 Girls 1 Cup bekannt wurde, Eingang in die öffentlichen sozialen Netzwerke.

Aufgrund ihrer Funktions- und Wirkweise lassen sich die Reaction Videos im Anschluss an Limor Shifman als (Reaction) Memes bezeichnen (vgl. Shifman 2011: 187-203). Das heißt, sie verbreiten sich digital durch Nachahmung und stoßen so immer neue Bildproduktionen an (vgl. ebd.: 10). Als Teil einer Serie werden die Videos mit einfachsten Mitteln produziert, um die Beteiligung möglichst niedrigschwellig zu gestalten, damit besonders viele User:innen an dem Reaction Meme teilhaben können. Auf diese Weise wird die ökonomische Strategie der Videoplattform unterstützt, weil immer mehr Daten für YouTube generiert werden.

Durch das Mitwirken an einer Serie gehören die Netzakteure zudem einem privilegierten Kreis von Individuen an, die sich gemeinsam zum verbreiteten Inhalt positionieren (vgl. Shifman 2014: 31). Obwohl Reaction Videos Massenphänomene sind, kann doch jeder einzelne Beitrag die eigene Kreativität und Einzigartigkeit unter Beweis stellen (vgl. Shifman 2011: 190). Durch das steigende Angebot solcher Videos wächst die Herausforderung an die Produzent:innen, einen Beitrag zu posten, der möglichst viel Aufmerksamkeit generiert.

Neben 2 Girls 1 Cup wurden weitere Videos bekannt, die für Reaction Videos genutzt wurden, etwa weitere Fetischpornos wie 1 Man 1 Jar oder 2 Kids 1 Sandbox. Die Titelgebung - die Verbindung aus Zahlen und Substantiven - ist zu einem zuverlässigen Marker für Bilder mit drastischem Inhalt geworden. Diesem Titelschema folgend wurden auch Videos wie 3 Guys i Hammer bekannt, in denen extreme physische Gewalt und sogar Mord gezeigt werden. ${ }^{1}$ Selbst zu den Enthauptungsvideos von Daniel Pearl und Nicolas Berg finden sich Reaction Videos. 
Videos wie die besprochenen, die Hardcore-Pornografie oder Gewalt zeigen, sind frei im Internet verfügbar. Sie werden als Mittel verwendet, um das jeweils als pervers und abnorm Verstandene einerseits in den öffentlichen Raum zu bringen und sich andererseits davon abzugrenzen und so $\mathrm{zu}$ positionieren. Der Umgang mit drastischen Bildern, die physische Gewalt, Tod und sexuelle Handlungen zeigen, ist also immer von der ambivalenten Spannung zwischen Faszination und Abstoßung geprägt.

Dieser Konflikt ist so alt wie die Tradition dieser Darstellungen selbst, so Susan Sontag. Drastische Bilder folgen weniger einem moralischen Nutzen, sie seien vielmehr Provokation und Herausforderung (vgl. Sontag 2003: 50). Besonders Fotografien erhöben den Anspruch, exakt das zu zeigen, was gewesen ist. Da die Betrachter:innen nicht in einer distanzierten Kontemplation verharren könnten, sondern in eine voyeuristische Position gerieten, schwinge in der Betrachtung von Fotografien mit drastischen Inhalten immer auch Beschämung mit (vgl. ebd.: 56). Den Betrachter:innen komme dann eine Teilverantwortung zu und sie würden herausgefordert, Stellung zu beziehen. Allein das Betrachten solcher Szenen der Herabsetzung, so lässt sich aus Sontags Ausführungen schließen, kann in uns also das Gefühl von Scham evozieren, weil wir im Moment des Schauens in Konflikt mit unserer Verantwortung als Zeug:innen (vgl. Klonk: 2017) geraten. In dem Moment, in dem uns in unserer voyeuristischen Position unser Genuss an den spektakulären Bildern bewusst wird, reflektieren wir unsere moralische Verantwortung gegenüber dem Gesehenen. Sontag räumt ein, dass längst nicht alle emotionalen Regungen beim Betrachten drastischer Bilder den Ansprüchen von Vernunft und Moral entsprechen. Allzu oft mischten sich zwischen Schock und Angst auch Interesse und Neugier.

Die Gefilmten, die sich - in Erwartung heftiger Gefühlsregungen - bewusst beim Betrachten drastischer Bilder filmen, um wiederum von einem Publikum gesehen zu werden, nehmen dann ihrerseits eine gewaltvolle Position gegenüber dem Gesehenen ein: Sie nutzen ihre Rolle als Zeug.innen aus, um Teil zu haben an einer digitalen Affektgemeinschaft (vgl. Schankweiler 2019). Ihr Blick auf die drastischen Szenen vor ihnen auf dem Bildschirm ist insofern ein herabsetzender Blick zum Zweck der Teilhabe. Ihre Schaulust hängt mit dem invektiven Blick erster Ordnung zusammen. Das Reaction Video besteht dann wiederum in der Verarbeitung des affektiven Überschusses, der aus dem invektiven Blick erster Ordnung resultiert. Es dokumentiert eine Reaktion, die vorher nur kalkuliert werden kann und oft genug auch die Überforderung der YouTuber:innen gegenüber dem Gesehenen festhält. In welcher Beziehung steht dieser invektive Blick erster Ordnung nun konkret zur angesprochenen Schaulust? 


\section{Von Schaulust zu Schauangst}

$\mathrm{Zu}$ Beginn von 3 guys 1 hammer reaction (2012) richtet sich die YouTuberin Kelcee Jane an ihre Zuschauer:innen (Abb. 4-5). Sie erklärt, sie werde sich gleich das Schockvideo 3 Guys 1 Hammer anschauen. Bereits vor der Aufnahme habe sie es gestartet und festgestellt, dass das Gesehene eine starke Reaktion bei ihr auslöst. Daraufhin habe sie entschieden, ihre Reaktion aufzuzeichnen. Die folgenden drei Minuten des Reaction Videos, in denen sie sich immer wieder die Augen zuhält, sich zwingt, doch hinzusehen und schließlich unter Tränen das Video abbricht, vermitteln den Eindruck einer Mutprobe, die sie sich selbst auferlegt hat. Schließlich warnt die YouTuberin weinend ihre Zuschauer:innen davor, sich das Video 3 Guys 1 Hammer anzuschauen und unterstreicht hierbei umso mehr ihre Leistung. Ähnliche ambivalente Reaktionen sind in zahlreichen anderen Reaction Videos zur besagten Vorlage zu beobachten. Die Reaktionen der Schauenden erzeugen mehr oder minder ostentativ den Eindruck, das bloße (freiwillige) Hinsehen sei eine schwer zu ertragende Zumutung. Dieses Darstellen eines Schauens gegen innere Widerstände haben die angesprochenen Reaction Videos zu 3 Guys 1 Hammer und 2 Girls 1 Cup gemeinsam, obwohl sich das Videomaterial inhaltlich grundlegend unterscheidet.

Abb. 4 und $A b b .5$
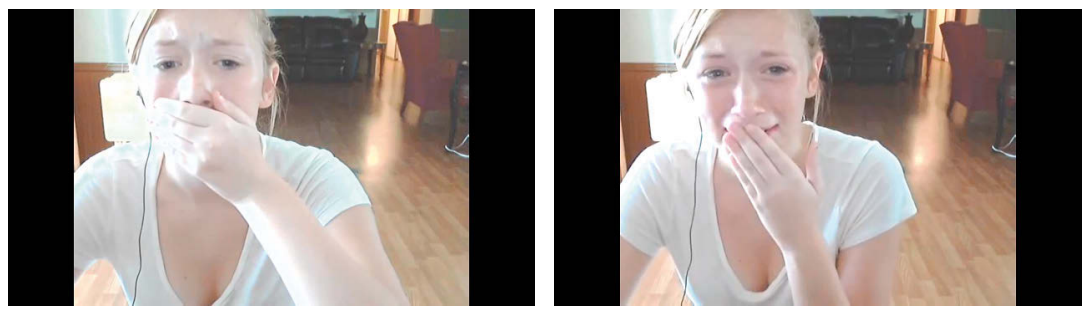

Die Ambivalenz zwischen Angst und Faszination angesichts drastischer Bilder schildert Georges Bataille lange vor der Erfindung digitaler Bilder. In Die Tränen des Eros [1961] beschreibt er die Fotografie der öffentlichen Folter des Chinesen Fu Zhu Li, der 1910 zum Tod durch Zerstückelung in hundert Teile verurteilt wurde (Abb. 6). Bataille hatte sie auf seinem Schreibtisch aufgestellt. Das Foto, so schreibt Bataille, drücke sowohl Ekstase als auch Schmerz aus. Und eben die Spannung zwischen diesen beiden Polen sei der Grund gewesen, weswegen ihn die Fotografie fesselte.

Das körperliche Leiden stellt sich Bataille als transfigurativen Akt vor. In ihm kann durch die bewusste Verschwendung des Lebens über den Kontrollverlust, der uns im Tod widerfährt, triumphiert werden (vgl. Bataille 1981: U2). Dem Leiden und dem Sterben zuzusehen mache uns zwar Angst, offenbare uns aber zugleich die "göttliche Ekstase« im Angesicht des Sterbens, das wir selbst nicht erleiden 
$A b b .6$

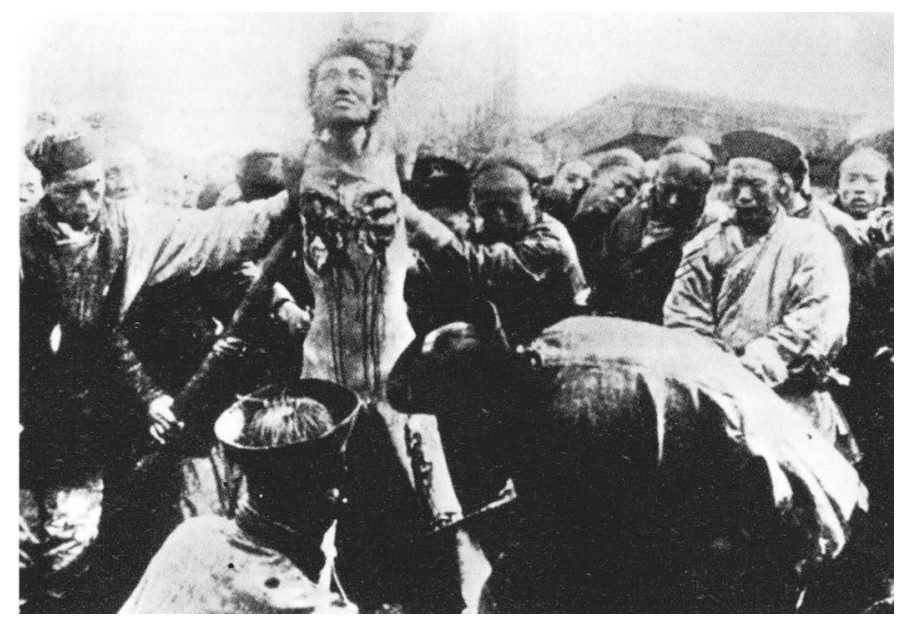

müssen. (Drastische) Bilder - das zeigt Batailles Beispiel - können mehr sein als das Abbild einer Realität. Indem sie uns die Erkenntnis über unsere eigene Verletzbarkeit vor Augen führen oder uns mittels einer Substitutionslogik vor dieser Verletzbarkeit bewahren sollen, können sie selbst Realitäten erschaffen. Sogar mehr noch: Nicht nur das Subjekt, sondern die Dinge selbst, besitzen die Fähigkeit, zurückzublicken.

Um die Wirkkraft der gewaltvollen oder sexualisierten Bilder zu beschreiben, die sich die YouTuber:innen in den Reaction Videos ansehen, lohnt sich eine Auseinandersetzung mit den Blickstrukturen, die die Relation zwischen Subjekt und Objekt beeinflussen. Maurice Merleau-Ponty etwa beschreibt das Sehen als ein Tasten, bei dem der Blick auf den taktilen Widerstand der Dinge trifft. Das Verhältnis zwischen Subjekt und Objekt ist so von wechselseitigen Blicken bestimmt (Merleau-Ponty 1986: 180f.). Ähnliches beschreibt Jacques Lacan, wenn er erklärt, dass das Subjekt nur von einem Punkt aus sehe, es aber »von überall her erblickt « werde (Lacan 1978: 78). Dadurch gerät das konstruierte Verhältnis von Subjekt und Objekt, Schauendem und Sichtbarem ins Wanken. Dieser Blick kann auch ein Geräusch sein, durch das sich das Subjekt angesprochen fühlt. Wenn etwa der Schlüssellochgucker ${ }^{2}$ nahende Schritte hört und in diesem Moment schamhaft seine Po-

2 Bei diesem Beispiel sowie den Ausführungen zur Verschränkung des Augen- und des Blicksehens in der Wechselwirkung zwischen Kunstproduktion und -rezeption verzichte ich auf die gendergerechte Schreibweise, weil ich mich hier auf Figuren beziehe, die als Begriffe in Sartres und Lacans Theorie geprägt sind. 
sition als Voyeur erkennt (Sartre 1991: 317), dann trifft ihn der imaginäre Blick und stellt ihn als begehrendes und damit mangelhaftes Subjekt bloß. Was ich also anschaue, das kann zurückschauen - unter Umständen mit vernichtender Wirkung.

Hier ist ein entscheidendes Moment der Wirkweise drastischer Bilder angesprochen, das einen zentralen Referenzpunkt für die Analyse der Produktionsmechanismen von Reaction Videos bildet. In der abendländischen Bildtradition ist die bedrohliche, sogar tödliche Wirkmacht des Bildes und die Angst vor ebendieser Macht immer schon Bestandteil der Rezeption gewesen. Vom Erstarren unter dem »bösen Blick« und vom davor schützenden Bild erzählt im westlichen Kulturkontext beispielhaft der Mythos der Medusa. Die griechische Legende berichtet von der Gorgone Medusa, deren Anblick so grauenerregend war, dass alle, die sie anschauten, zu Stein erstarrten. Erst Perseus konnte die Gorgone besiegen, indem er sie enthauptete. Vermittelt über ihr Spiegelbild in einem blanken Schild konnte Perseus die Medusa ansehen, ohne zu versteinern.

Diese Geschichte greift Linda Hentschel 2008 für ihre Überlegungen zur Bilderpolitik in Zeiten von Krieg und Terror auf. Sie liest im Mythos des Perseus, der sich einem Bild anvertraut, um die grausame Realität zu besiegen, eine Immunisierungslogik, bei der das Subjekt durch den Anblick drastischer Bilder in homöopathischen Dosen einen Schutz gegen die tatsächliche Grausamkeit der Realität aufbauen kann. Die Schauenden empfänden beim Anblick der Bilder zwar starke emotionale Regungen, doch immer im Bewusstsein, selbst nicht in Gefahr zu sein (vgl. Hentschel 2008: 186). ${ }^{3}$

Hentschels Ausführungen sind an dieser Stelle hilfreich, um zu verstehen, wie das Betrachten drastischer Bilder zur Bewältigungsstrategie einer schreckenerregenden Realität werden kann. Für Hentschel ist nämlich das Betrachten dieser Bilder eine Selbstbestätigung der eigenen Sicherheit. Der Drang zum Schauen, den die Gefilmten in den Reaction Videos trotz Ekel und Schock zu empfinden scheinen, kann - folgt man dieser Logik - auf den regulativen Effekt der Desensibilisierung zurückgeführt werden. Dem Schaudrang läge also eben diese rettende Wirkung der angstbesetzten Bilder zugrunde.

Diese Bilderpolitik beruhe, so Hentschel, auf dem Abgrenzungsmechanismus gegen als fremd und gefahrvoll Wahrgenommenes zugunsten der eigenen Identitätsbildung (vgl. ebd.: 195). Insbesondere in Zeiten großer Verunsicherung durch 
Krieg und Terror bediene dieser Bildumgang verstärkt traditionelle Repräsentationssysteme (vgl. ebd.: 194). So zeige man etwa seine eigenen Toten nicht, während die Toten der anderen als Trophäen ausgestellt würden (vgl. ebd.: 195f.). Wie das Spiegelbild der Medusa Perseus' Rettung bedeute, fungiere auch das Bild des anderen Todes als »Trophäe des eigenen Überlebens« (ebd.: 195f.). Die drastischen Bilder förderten in uns den verbotenen Wunsch zu Tage, das Leben anderer gefährdet zu sehen, um die eigene Sicherheit zu imaginieren. Hier zeigt sich erneut das Potenzial des invektiven Blickes erster Ordnung, der die Herabsetzung eines anderen Lebens zu Gunsten des eigenen Sicherheitsgefühls einfordert.

Indem die Gefilmten ihre abwehrende Reaktion gegenüber den drastischen Bildern von 2 Girls 1 Cup und 3 Guys 1 Hammer öffentlich zeigen, negieren sie performativ jede Verbindung zur gesehenen Grenzüberschreitung und dokumentieren das vor Publikum. Die Bilder der Videos 2 Girls 1 Cup und 3 Guys 1 Hammer verstoßen nicht nur gegen geltende Moralvorstellung, sondern zeigen im Fall von 3 Guys 1 Hammer sogar schwerwiegende Straftaten. Die Bilder lassen das schauende Individuum zurückschrecken. Dennoch bleiben die YouTuber:innen meist vor den Bildschirmen sitzen und schauen weiter auf den Monitor. Das Schauen-Müssen lässt sich einerseits mit dem Wettbewerb um die größte Aufmerksamkeit im Internet begründen. Verstanden als Konfrontation mit dem medusischen Bildblick dient die mediale Praktik des Nicht-Wegschauen-Könnens in den Reaction Videos aber zugleich als eine kollektive Strategie, sich der eigenen sicheren, zivilisierten und integren Position zu versichern.

Konstitutiv für die moralische Überlegenheit ist dabei, dass die YouTuber:innen ihre Reaktionen und verurteilende Haltung gegenüber dem Gesehenen vor einer Netzöffentlichkeit inszenieren. In den Reaction Videos verurteilen sie Grausamkeit, Perversion und moralische Fragwürdigkeit vor einem Publikum und leugnen somit zugleich durch ihre teils stark ausgestellte abwehrende Haltung, die Existenz solcher Eigenschaften in ihnen selbst. Sie machen diese »Eigenschaften zum anderen ihrer selbst « (Butler 2005: 59), sondern sie aus und markieren sie als das Grausame und Perverse, das zu einem anderen gehört.

\section{Ekel als definitorisches Mittel}

So vielfältig die Formen von Reaction Videos zu drastischen Bewegtbildern sind, so einförmig sind die Reaktionen, die die YouTuber:innen zeigen; fast immer spiegeln sich auf den Gesichtern Reaktionen der Ablehnung und des Ekels. Was auf dem Bildschirm sichtbar ist, wird durch die Reaktionen der Personen vor der Kamera klar als abnorm und pervers gekennzeichnet. So löst der Anblick der Protagonistinnen von 2 Girls 1 Cup, wie sie mit den Körperausscheidungen in Berührung kommen, bei den YouTuber:innen heftigsten Ekel aus. Kot, Urin und verwesende 
Materie sind Stoffe, die in den allermeisten Fällen Abneigung, Abscheu und Ekel provozieren. Ekel hat den Schutz und die Selbstbehauptung des Individuums zum Ziel und unterliegt komplexen gesellschaftlichen Konstruktionsprozessen.

Die Frage nach dem Ursprung der Verwerfung durch Abwehrreaktionen wie dem Ekel fokussiert auch Julia Kristevas Abjekt-Theorie. Zugleich bietet sie einen Erklärungsansatz sowohl für die heftige Reaktion auf bestimmte Substanzen als auch auf Handlungen wie Straftaten. Ihre Untersuchung führt das Bedürfnis nach Abwehr und Abgrenzung zugleich auf spezifische Reaktionen zurück, die auch in den beschriebenen Reaction Videos auftreten. Als Beginn der Subjektwerdung oszilliert für Kristeva der Begriff des Abjekten zwischen dem Todestrieb, der das Subjekt mit dem Leib der archaischen Mutter wiedervereinen möchte, und dem Willen zum Subjektwerden, der diesen mütterlichen Körper als das »Abjekt« abspaltet. Der abjekte mütterliche Körper, der dem Subjekt seine ursprüngliche und jetzt verworfene Abhängigkeit vor Augen führt, müsse geleugnet werden (vgl. Kristeva 1982: 1-31). Noch vor dem Eintritt in die symbolische Ordnung habe das Subjekt eine Einheit mit diesem Körper gebildet. Im Dienst der Subjektwerdung müsse sich das Individuum von allem, was an seinen früheren Zustand erinnert, abgrenzen. So werde der mütterliche Körper als Abjekt verworfen. Das Subjekt leugne auf diese Weise sein eigenes (Nicht-)Sein im mütterlichen Körper (vgl. Menninghaus 2002: 529). Dieser Körper ist darum nicht nur in seiner biologischen Substanz zu begreifen, sondern auch in seinem Verhältnis zur symbolischen Ordnung. Denn gerade, indem das Subjekt ihn als Abjekt verwerfe, sei er Vorbedingung von Sprache (vgl. ebd.: 75-85).

Abb. 7 und Abb. 8
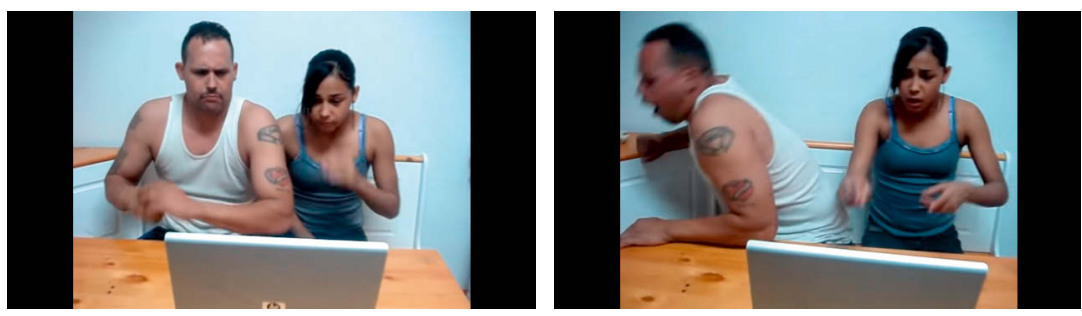

In Kristevas Perspektive werde nicht nur die Existenz vor dem Subjektwerden im mütterlichen Körper, sondern auch das Ende jedes Subjekts - der Tod geleugnet. Die verwesende Leiche sei etwa abjekt, weil sie buchstäblich aus der symbolischen Ordnung falle, durch die das Subjekt sich selbst aufrechterhalte. Sie mache deutlich, dass das Leben immer schon mit dem Tod infiziert ist (vgl. Kristeva 1982: 4). Körperflüssigkeiten und -ausscheidungen, wie sie im Trailer 2 Girls 1 Cup 
ausgestellt werden, sind abjekt, weil sie ebenso Ausdruck des sterblichen Körpers sind. Der Umgang der Darstellerinnen mit jenen Körperausscheidungen konfrontiert das schauende Subjekt mit dem eigenen verdrängten Begehren. Zu sehen, wie andere lustvoll mit dem Objekt des Begehrens umgehen, das ich mir selbst verbiete, muss in mir den Abjektionsekel auslösen. Denn hiermit wird die fragile Grenze geschützt, die ich als Subjekt um mich errichtet habe. ${ }^{4}$ Das Abjekt bekräftigt zum einen die ursprüngliche Verwerfung, durch die es erst entsteht, und bestätigt zum anderen seine Kontinuität im Bewusstsein des Subjekts. So sei jede Abjektion in Wirklichkeit die Anerkennung des Mangels, der durch die ursprüngliche Verwerfung des mütterlichen Körpers ausgelöst werde (vgl. Menninghaus 2002: 529).

Abb. 9 und Abb. 10
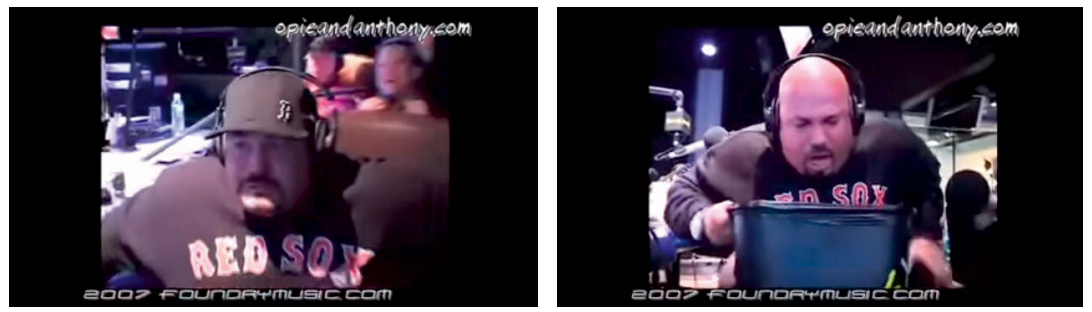

Da Kristeva innerhalb des psychoanalytischen Modells argumentiert, ist der Ekel der Abjektion nicht an rein biologische Reflexe gebunden, auch wenn er sich besonders in den beschriebenen Reaction Videos als heftige körperliche Reaktion ausdrückt. Vielmehr ist er eine gesellschaftlich vermittelte Reaktionsweise (vgl. Kristeva 1982: 10). Der Ekel gegenüber dem Abjekt wird auch deswegen ausgelöst, weil das Abjekt Verbote außer Kraft setzt (vgl. ebd.: 15.). Insofern werden nicht nur Substanzen als abjekt verworfen, sondern auch Verhaltensweisen, die gesellschaftliche Grenzen verletzen (vgl. ebd.: 4). Gewalt und Mord, wie sie auch in 3 Guys 1 Hammer gezeigt werden, verstoßen in besonders drastischer Weise gegen diese Grenzen. Im Vergleich zu den Reaktionen in den 2 Girls 1 Cup Reaction Videos zeigen die YouTuber:innen beim Anblick des Snuff-Films vor allem Betroffenheit und Schock als eine Form der abgrenzenden und distanzierenden Haltung. Ekel ist hier seltener zu sehen, gehört aber ebenso zum Spektrum der artikulierten Verhaltensweisen.

In dem Video 3 Guys 1 Hammer Reaction (wtfitskelsey 2009) (Abb. 11-12), in dem sich drei junge Frauen dabei filmen, wie sie sich den Snuff-Film ansehen, drücken

4 Wenn sich eine:r der Gefilmten in 2 girls and 1 cup reaction [daddy and Ashley] =] (Abb. 7-8) oder 2 Girls 1 Cup Reaction - Robert Kelly (Abb. 9-10) beim Anblick des Videos sogar erbricht, dann ist das die buchstäblichste Form der Abjektion. 
die YouTuberinnen ihren Ekel durch heftiges Würgen und Husten aus, mehrfach bezeichnen sie das Gesehene als »disgusting«. Den versehrten Körper des Mannes im Video zu sehen, seine Verletzungen und schließlich sein Sterben zu beobachten, löst bei den jungen Frauen Ekel aus. Mit dem Ekel drücken sie aber nicht nur Abwehr gegenüber der Gewalt und dem Mord aus, sondern auch gegenüber der Versehrtheit des Sterbenden. Durch ihre Haltung wird die Ordnung, innerhalb derer sich das Subjekt definiert, aufrechterhalten. Der Ekel ist bei Kristeva also eine Abgrenzung, die sowohl die reinen gesellschaftlichen Strukturen des Subjekts als auch das Phantasma seines reinen Körpers wahren soll.

Abb. 11 und Abb. 12
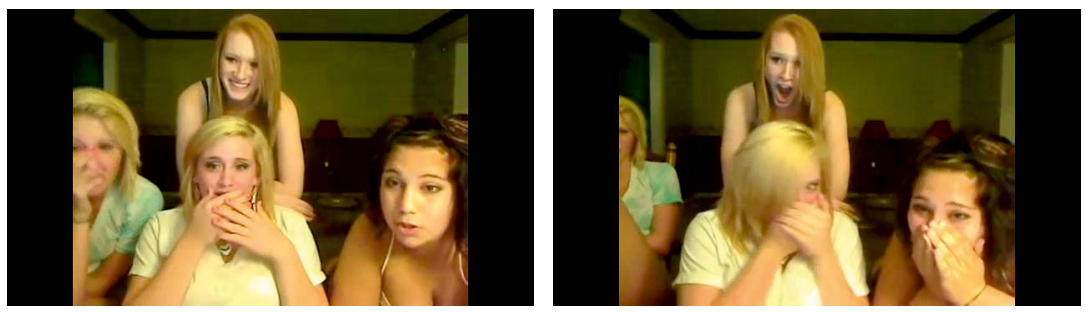

In theoriegeschichtlicher Perspektive muss Kristevas Theorie kritisch entgegengesetzt werden, dass die Bezeichnung des mütterlichen Köpers als Abjekt nur aus einer patriarchalen Gesellschaftsstruktur heraus denkbar ist, in welcher der weibliche Körper auf seine Gebärfähigkeit reduziert wird. Erst der mütterliche Körper macht die menschliche Existenz möglich und ist die elementare Funktionsstelle der Reproduktion, daher muss er im patriarchalen System verworfen und zum Abjekt erklärt werden.

Bezieht man dieses Argument auf die Digitalität der Reaction Videos, wird der menschliche Körper und seine Affekte zur letzten ausbeutbaren Ressource eines Kapitalismus, der sich ständig durch Ressourcenknappheit bedroht sieht. Um diese existenzielle Funktion des affektiven Körpers zu invisibilisieren, muss auch er als Abjekt verworfen werden. Die Abjektion ist so ein zentraler Bestandteil der Reaction Videos. Sie stoßen eine unendliche Kette affektiver Kommunikationshandlungen an und legen so wiederum unerschöpfliche Ressourcen offen.

Eine weitere Form der Verwerfung neben dem Ekel ist das Lachen. In Two Girls One Cup Reaction Video!!! LMAO!!! springen etwa drei Jungen immer wieder von ihren Stühlen auf und halten sich Tränen lachend die Hände vor den Mund (vgl. Racardo Davis 2010) (Abb. 13-14). Das heftige Lachen der Gefilmten steckt buchstäblich zum Mitlachen an. Während der Affekt des Ekels eine eindeutig ablehnende Empfindung ausdrückt, kann Lachen durch unterschiedliche Emotionen ausgelöst werden. Bei Kristeva steht das Lachen insbesondere als Verlachen mit der Abjek- 
tion in enger Beziehung. Lachen ist dann zwar zunächst ein Akt des Ausschlusses und ähnelt so dem Erbrechen durch Ekel, bietet aber zugleich in der plötzlichen Entladung einer Spannung die Möglichkeit, Kontakt mit dem Abjekt aufzunehmen (vgl. Kristeva 1982: 8). Das Lachen ist also ein Mittel, mit dem Ekel umzugehen, eine affektive Reaktion auf den Ekel und ein Ausdruck für diesen. Es ermöglicht dem Subjekt, das Abjekt zu inkorporieren und in der Geste des Lachens wieder auszustoßen. Auf diese Weise enthält das Lachen das Potenzial, »Verbotsschranken [zu heben], [...] in das Verbotene [einzudringen] und den zerstörerischen, gewaltsamen, befreienden Trieb in es [hinein zu tragen] « (Kristeva 1978: 218). So bietet das Lachen die Möglichkeit, sich in diesem Affekt von gesellschaftlichen Zwängen zu entlasten (vgl. dazu: Freud 1958). Doch auch wenn das Lachen einen Kontakt mit dem Abjekt zulässt, stößt es dieses zuletzt wieder aus. Dieser ambivalente Charakter des Lachens ist möglicherweise der Grund, warum es mitunter als beleidigend oder grausam empfunden werden kann. Was in den 2 Girls 1 Cup Reaction Videos als höhnisches Verlachen der als eklig empfundenen Handlungen der Protagonistinnen im Trailer akzeptiert wird, erzeugt in den 3 Guys 1 Hammer Reaction Videos jedoch den Eindruck, die Lachenden machten sich über das Opfer lustig und bezeugten somit ihre Komplizenschaft mit den Tätern. Das Lachen wirkt oft viel weniger kontrolliert, mehr wie eine emotionale Überforderung. Vor dem Hintergrund von Kristevas Theorie positionieren sich die Schauenden auf diese Weise auch zu dem Verbrechen, das im Video gezeigt wird. Bei 3 Guys 1 Hammer Reaction verhalten sich die jungen Frauen zwar ablehnend gegenüber dem Gesehenen, unterstützten es aber gleichzeitig durch den Schaudrang, dem sie nachgeben - sie schauen eben zu. So ist die Schaulust hier auch eine Form der Zustimmung, eine Komplizenschaft, die sie mit den Tätern eingehen. Im lustvoll-affirmierenden Zuschauen nehmen die Schauenden zum Gesehenen eben jene, oben beschriebene invektive Blickposition ein.

Abb. 13 und Abb. 14
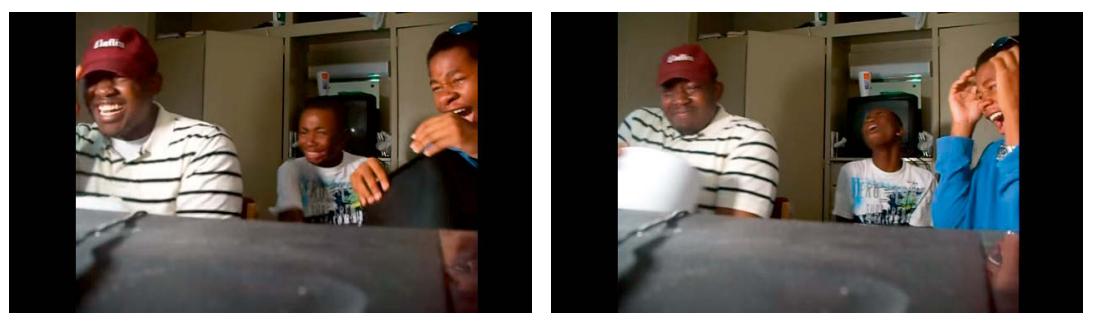

Es ist verblüffend, dass das Selbstexperiment - sich öffentlich beim bewussten und freiwilligen Schauen drastischer Bilder auszustellen - ständig wiederholt 
wird, ist doch jedes Mal erneut eine negative Bewertung durch die Netzgemeinschaft zu befürchten. Es scheint, als diene das Reaction Video als Mittel, die Kontrollierbarkeit der eigenen Reaktion vor einem bewertenden Publikum zu testen. Es wird so selbst zu einer Form der medialen Kontrolle über das Unkontrollierbare.

\section{Der invektive Blick zweiter Ordnung}

Im beschriebenen Beispiel von 3 Guys 1 Hammer Reaction, in dem sich drei junge Frauen filmen, lachen die Gefilmten zu Beginn des Videos besonders laut. Die Frauen kommentieren ihre Verhaltensweisen immer wieder gegenseitig und thematisieren auch die Frage nach richtigem oder falschem Verhalten. Obwohl eine der jungen Frauen von Beginn des Videos an immer wieder laut auflacht und kichert, verurteilt sie dieses Verhalten bei den anderen. Ihr eigenes Lachen scheint sie nicht zu reflektieren oder als unpassende Verhaltensweise wahrzunehmen.

Auch am Schluss der oben erwähnten 3 guys I hammer reaction thematisiert die YouTuberin Kelcee Jane ihr eigenes Verhalten, indem sie sich an ihre Zuschauer:innen wendet: "Don't watch - I am kind of laughing right now, scause I'm crying." Dass sie lache, weil sie weine, eine zunächst wenig plausible Erklärung, mag jedoch ihre ambivalente Reaktion auf ihre emotionale Überforderung beschreiben. Der Reflex, das eigene Lachen oder das Lachen der anderen als unpassende Reaktion zu reflektieren, zeigt, dass ein Bewusstsein über die zweite Beobachtungsebene existiert, von der aus die imaginierten Betrachter:innen zusehen.

Von dieser Beobachtungsebene wird auch eine beurteilend-moralische Haltung zu den im Reaction Video gezeigten Verhaltensweisen erwartet bzw. imaginiert, wenn die Gefilmten ihre eigene Reaktion rechtfertigen oder die Reaktion anderer aus der Gruppe reglementieren. Zusätzlich zu dieser imaginierten Beobachtungsinstanz werden die YouTuber:innen aber auch mit der konkreten und reellen Position anderer Netzakteure konfrontiert, wenn die Reaction Videos durch die Mittel der phatischen Kommunikation Aufmerksamkeit bekommen.

Das digitale Subjekt ist im Prozess der Subjektwerdung mit zwei Größen konfrontiert. Zum einen sieht es sich dem »verallgemeinerten Anderen« gegenüber, einer Kategorie, die gemeinsame Wertesysteme repräsentiert, vor der das Individuum die eigenen Handlungen immer wieder reflektiert und so das "Ich« formt (vgl. Schachtner/Duller 2014: 85). ${ }^{5}$ Zum anderen wird das digitale Subjekt in der Interaktion mit anderen Netzakteuren vom Blick des »konkreten Anderen« getroffen

5 Schachtner und Duller beziehen sich hier primär auf George H. Meads Ausführungen zur Konstitution des Subjekts in Gemeinschaften. Vgl. dazu: Mead 1973. 
(vgl. ebd.: 87f.). ${ }^{6}$ Dieser Blick vermittelt sich nicht face-to-face, sondern über die Social Signals, die YouTube zur Verfügung stellt - positiv durch Likes und ChannelAbonnements oder negativ durch Dislikes und Sanktionierungen ${ }^{7}$. Das Individuum, das sich selbst nur in Teilen wahrnehmen kann, wird von diesem Blick in seiner Ganzheit gespiegelt. Es sind sowohl die im Inneren des Subjekts stattfindenden Konflikte, die durch den verallgemeinerten Anderen ausgelöst werden, als auch die von außen auf es gerichteten Blicke der konkreten anderen, die das Subjekt zum Subjekt machen (vgl. ebd.: 85-88).

Zwischen der Instanz des verallgemeinerten und der Kategorie des konkreten Anderen gilt es für die Analyse der Reaction Videos eine weitere Figur zu positionieren, die ich den:die imaginierte:n Zuschauer:in nenne. Erst dann kann nämlich die spezifische digitale Subjektkonstitution in der Produktion von Reaction Videos in den Blick genommen werden. Diese imaginierte Beobachtungsinstanz repräsentiert das digitale Publikum, das immer auch eine latente Bedrohung darstellt, weil es für das im Internet für alle sichtbar handelnde Subjekt unsichtbar und so nicht abschätzbar ist. Gelingt die Selbstinszenierung des Subjekts, kann sich der:die imaginierte Zuschauer:in als begeisterter Fan manifestieren. Es kann aber auch passieren, dass ein digitaler Beitrag auf zahlreiche Hater trifft. Das digitale Subjekt muss sich also ständig mit diesen mannigfaltigen möglichen Reaktionen des Publikums auseinandersetzen. Auch, wenn die Gefilmten ihre Reaktionen nicht im Reaction Video thematisieren, sind sie sich doch immer dessen bewusst, dass sie angeblickt werden. Sobald ein Subjekt im digitalen Raum aktiv wird, ist es den Blicken des konkreten Anderen ausgesetzt.

Die Bewertungen werden durch den konkreten Anderen - die rezipierenden Netzakteure - vorgenommen. Im Fall der Negativbewertung kann der Blick der:des imaginierte:n Zuschauer:in ein herabsetzender sein. Hier offenbart sich der invektive Blick zweiter Ordnung, dessen beschämende Wirkung nicht mehr auf die drastischen Bilder abzielt, sondern auf die Reaktionsweisen der Gefilmten, die sich zu diesen Bildern verhalten. Diese Herabsetzung kann insbesondere in den Kommentaren extreme Ausmaße annehmen, weil die Sozialen Medien affektive Kommunikation beschleunigen und die User:innen durch ihren phatischen Charakter kommunikativ an sich binden. Gegenüber dem Subjekt, das sich innerhalb der Reaction Videos inszeniert, treten die Rezipierenden als konkrete Andere auf. In dem Moment, in dem sie ihre Rezeption durch Kommentare produktiv machen, sind sie aber ihrerseits mit verallgemeinertem Anderen und konkretem Anderen konfrontiert. Mit dem Perspektivwechsel von der Produktions- auf die Rezeptionsebene stitution durch den unmittelbaren Blick eines Anderen. Vgl. dazu: Meyer-Drawe 1990. 
verändert sich auch die Beobachtungsebene von der unmittelbaren ersten Beobachtung zur zweiten Beobachtung, die sich durch die Reaktion der Schauenden vermittelt. Dabei wechseln auch die Verhältnisse von Sichtbarkeit und Unsichtbarkeit, da die Bildquelle des drastischen Videos nun nicht mehr Bestandteil der unmittelbaren Wahrnehmung ist. Im Zentrum stehen nun allein die Reaktionen auf diese Bilder. Die Betrachter:innen der Reaction Videos schauen ausschließlich $\mathrm{zu}$, wie jemand zuschaut.

\section{Unendliche Beobachtungsebenen und produktive Rezeption}

Solange sich die Rezeption der Reaction Videos im Interface von YouTube abspielt, begünstigen die Social Signals eine tendenziell bewertende Rezeptionshaltung. Hier bewegen sich die Handlungsmöglichkeiten der Rezipient:innen noch immer in der spezifischen Aufmerksamkeitsökonomie (Franck 1998) des digitalen Raumes. Die Handlungsmöglichkeiten sind durch die von YouTube bereitgestellten Funktionen, die Aufmerksamkeit quantifizierbar machen sollen (Mau 2018: 24), weitgehend gesetzt. Eine Auseinandersetzung oder Reflexion der YouTuber:innen über die Möglichkeiten der Beobachtung jenseits der zweiten Beobachtungsebene in den Kommentarspalten hinaus findet hier selten bis gar nicht statt. Dennoch bilden sich auch Strategien einer produktiven Rezeption heraus, die eine dritte Beobachtungsebene mit einbeziehen. So finden sich Reaction Videos, die ihrerseits auf Reaction Videos anderer, meist bekannterer YouTuber:innen reagieren. Auch hier wird deutlich, wie stark die Produktion der Reaction Reaction Videos zur Akkumulierung von Aufmerksamkeit und somit auch ökonomischen Kapitals verwendet wird. Die YouTuber:innen nutzen die Reichweite der erfolgreicheren Internetstars, um ihre eigene Position innerhalb der digitalen Aufmerksamkeitsökonomie zu verbessern. Die kreative Auseinandersetzung mit den vorhandenen Reaction Videos bringt hier eine dritte Beobachtungsebene ins Spiel, die bis ins Unendliche vervielfacht werden kann.

Ein Beispiel, wie die produktive Rezeption sich in eine unendliche Kette von Beobachtungsebenen verwandeln kann, ist das Video Reacting To The Reaction Of The Reaction To The Reaction Of The Reaction Of The Reaction To The Reac (MrBeast: 2017), bei dem sich ein YouTuber immer wieder dabei filmt, wie er selbst auf eine Rede des ehemaligen US-amerikanischen Präsidenten Donald Trump und in der Folge jeweils auf seine eigenen Reaktionen reagiert. Am Ende entsteht ein Zusammenschnitt einer 20-fachen Reaktion des YouTubers. Das Video der Rede Trumps als Auslöser für die erste Reaktion gerät innerhalb der Reaktionskette immer weiter aus dem Blickfeld. Dieses Beispiel ist durch die Offenlegung der unendlichen Reaktionsketten als Kommentar auf das Internetphänomen der Reaction Videos lesbar. 
Solche Beiträge führen ihre eigene Logik und Wirkweise vor, bleiben aber weiterhin dem Kontext von YouTube verhaftet.

Wenn dem Umfeld der Reaction Videos die Bewertungs- und Quantifizierungsmittel entzogen werden, indem sie außerhalb des Kontextes von YouTube situiert werden, hat das einen Einfluss auf Rezeption und Wirkweise. Beispielhaft zeigt das die künstlerische Videoarbeit Emilys Video des Künstler:innenpaares Eva und Franco Mattes, in der sich das Duo unter anderem mit viralen Internetphänomenen, wie etwa dem Hype um Reaction Videos zu Schockvideos, auseinandersetzen. Emilys Video zeigt über eine Webcam aufgezeichnete Personen, die sich freiwillig dabei filmen ließen, wie sie sich einen für die Zuschauer:innen von Emilys Video nicht sichtbaren Film auf einem Laptop anschauen. Der Film war ein Zusammenschnitt verschiedener Clips, die aus dem Darknet stammten, und wurde anschließend durch das Künstler:innenduo zerstört. Die Reaction Videos weisen das von YouTube bekannte Setting auf: die verwaschene Ästhetik der Webcam, herkömmliche Einrichtungsgegenstände in privaten Wohnungen im Hintergrund der Szenerie. Obwohl das Video, das sich die Gefilmten anschauen, angeblich immer dasselbe ist, fallen die Reaktionen, wie schon in den Reaction Videos zu 2 Girls 1 Cup und 3 Guys 1 Hammer, sehr vielfältig aus. ${ }^{8}$ Viele der Betrachtenden schauen betroffen auf den Bildschirm, manche halten sich die Augen zu oder verlassen sogar den Raum. Manche lächeln still, kneifen dann aber die Augen zusammen. Wieder andere lachen laut und beugen sich interessiert vor oder kommentieren das Gesehene.

Für die Installation im Ausstellungskontext 2012 in der Galerie Carrol/Fletcher in London wurden die einzelnen Reaction Videos hintereinander geschnitten und als Abfolge auf einem freistehenden Monitor abgespielt. Der Monitor war hochkant aufgestellt und leicht nach hinten geneigt. Das Reaction Video nahm nur das obere Drittel der Bildfläche ein. Der Rest des Monitors war schwarz gefärbt, sodass sich die Besucher:innen der Ausstellung, die sich vor dem Monitor platziert hatten, darin spiegelten und sich somit selbst beim Betrachten der Reaktionen der gefilmten Personen betrachten konnten. An dieser Stelle schließt sich der Kreis der beobachtenden Beobachtung bei der Selbstbetrachtung der Rezipient:innen - und verlässt zugleich den Raum des Digitalen.

Diese von den Mattes' gefilmten Reaction Videos erinnern selbst stark an die klassischen YouTube Reaction Videos. Außerhalb des digitalen Raumes sind die Videos allerdings der phatischen Kommunikationszirkulation enthoben. Zudem ist

8 Was als Hintergrundmusik in den Emilys Video Reactions zu hören ist, ist laut einem YouTubeKommentar das Theme aus Werner Herzogs Aguirre, der Zorn Cottes. Vgl. fuckingharpsichord: https://www.youtube.com/watch?v=DDvNPoREzho [letzter Zugriff: 19.01.2021]. Teilweise sind aber auch Geräusche aus den Computerlautsprechern zu hören, die aus 3 Cuys 1 Hammer stammen, es ist also anzunehmen, dass dieses Video für den Zusammenschnitt verwendet worden ist. 
das Video, das sich die Gefilmten in den Emilys Video Reactions ansehen, im Gegensatz zu den 2 Girls 1 Cup und 3 Guys 1 Hammer Reactions, tatsächlich zur Leerstelle geworden, da es zerstört wurde. Den Reaktionsweisen der Gefilmten kommt so gesteigerte Aufmerksamkeit $\mathrm{zu}$, da sie die einzigen Hinweise auf den Inhalt des Videos geben. Die neue Umgebung, in der die Reaction Videos situiert sind, verhindert in doppelter Weise eine moralisch-bewertende Rezeptionshaltung in Bezug auf die Reaktionsweisen der Gefilmten: Einerseits, weil die Rezipient:innen den Grund für den Auslöser nicht nachvollziehen können. Andererseits, weil ihnen keine technischen Instrumente an die Hand gegeben werden, um ihre Reaktionen $\mathrm{zu}$ dokumentieren.

So verlangen die Emilys Video Reactions eine andere Rezeptionshaltung, die unmittelbar durch die Positionierung des spiegelnden Monitors eingefordert wird. Wo sich das rezipierende Subjekt vorher im Privaten von den heftigen Reaktionen der Gefilmten der Reaction Videos unterhalten ließ, wird es nun in einem öffentlichen Ausstellungsraum mit diesen Bildern konfrontiert und dabei selbst ausgestellt. Die Reaktionen der Rezipierenden werden auf YouTube erst durch die Mittel der Quantifizierbarkeit sichtbar, im Ausstellungsraum dagegen erfolgt die Reaktion direkt vor den Augen der konkreten Anderen - und vor dem Subjekt selbst. Durch den reflektierenden Monitor wird den Betrachtenden buchstäblich ein Spiegel vorgehalten.

Der bewertenden Rezeption in den sozialen Medien wird hier eine Subjektivierungsform gegenübergestellt, bei welcher der Blick reflexiv auf die Betrachtenden zurückgeworfen wird. Die Reaction Videos zu Emilys Video finden dennoch ihren Weg aus dem Offline- zurück in den Onlinebereich, denn sie sind als Playlist auf YouTube verfügbar. Hier zeigt sich aufs Neue, wie zentral die akkumulierte Aufmerksamkeit eines Beitrags für die Bewertung durch Social Signals ist.

Der Transfer der von YouTube bekannten Reaction Videos in den analogen Kontext eines Ausstellungsraumes zeigt, dass das Reaction Video zu einem Topos geworden ist, der sich unabhängig von seinem ursprünglichen Bezugsrahmen zitieren lässt. Ein weiteres Beispiel dafür ist 2 Girls 1 Cup - Ü55 Reaction Video - NEO MAGAZIN mit Jan Böhmermann in ZDFneo (ZDFneo: 2014). Das Video wurde 2014 im Rahmen der Satire-Show Neo Magazin Royale im Zweiten Deutschen Fernsehen (ZDF) produziert.

Nach einem eingeblendeten Ankündigungstext erklingt die bekannte Hintergrundmusik »Lover's Theme« aus 2 Girls I Cup. In Großaufnahme wird das Gesicht des Moderators Jan Böhmermann sichtbar, hinter ihm auf der Leinwand scheint verschwommen, weil außerhalb des Kamerafokus, der Trailer abzulaufen. Im Hintergrund sind neben der Musik Rufe der Überraschung und der Ablehnung zu hören. Die eingeblendeten Zuschauer:innen - Frauen und Männer etwa zwischen 50 und 70 Jahren - schauen gequält an der Kamera vorbei auf die Leinwand, manche lachen, andere verziehen angeekelt das Gesicht oder halten die Hand vor die 
Augen. Schließlich wird der Hinweis eingeblendet, das eben durchgeführte Experiment diene nicht der Unterhaltung, sondern ausschließlich der Marktforschung. Damit wird das ökonomische System, in dem sich die Reaction Videos verorten, transparent gemacht, denn in ihrem Hintergrund laufen Algorithmen der Vermarktung von Daten ab. Zudem spielt die Parodie mit den Klischees eines älteren Fernsehpublikums des öffentlich-rechtlichen ZDF und junger Internetuser:innen. Die drastischen Bilder des Scat-Porns sowie ihre Bearbeitung durch Reaction Videos, so die überspitze Aussage des Satirebeitrags, seien es, womit sich junge Internetnutzer:innen tagtäglich konfrontierten. Das Reaction Video selbst wird dann vom Studiopublikum des Neo Magazin Royal, vom Fernsehpublikum der Satireshow und letztlich wieder von Netzakteuren im digitalen Kontext rezipiert. Denn auch dieses Video ist aufYouTube verfügbar. Dort wird es - wie schon die einzelnen Reaction Videos von Emilys Video - erneut in die Aufmerksamkeitslogik der Plattform eingespeist. Als Teil einer TV-Sendung diente das aus dem Digitalen stammende Reaction Video im Neo Magazin Royale als ironischer Kommentar auf das Verhältnis zwischen sozialen Medien und älteren Massenmedien sowie den damit verbundenen Klischees.

Fraglich bleibt, ob die Zuschauer:innen im Fernsehstudio tatsächlich auf 2 Girls 1 Cup reagieren oder ob es sich hier nur um einen Zusammenschnitt handelt, der diesen Eindruck erzeugt. Wenn die Kamera die Großaufnahme Böhmermanns zeigt, werden hinter ihm auf der Leinwand verschwommen Bilder sichtbar, die durchaus aus dem Schockvideo stammen könnten. Die Rezipient:innen des Reaction Videos allerdings bleiben im Ungewissen darüber, ob diese Großaufnahme später mit Aufnahmen des Studiopublikums kombiniert worden ist, das möglicherweise auf etwas ganz anderes reagiert. So bleibt das Bild, auf das die Gefilmten reagieren, vor allem der Imagination der Rezipierenden des Reaction Videos überlassen.

Hier ist ein zentraler Punkt der Vermittlung einer »authentischen" Reaktion angesprochen, der sich auch aus den Produktionsbedingungen der Reaction Videos ergibt. Die user:innengenerierten Reaction Videos sind technisch einfach zu produzieren. Es handelt sich um One-Shot-Aufnahmen in geringer Auflösung, die meist keine Postproduktion durchlaufen. Auf Ebene der Rezipienten:innen stiftet diese Produktionsweise einen Bezugsrahmen, Wiedererkennung und auch Authentizität.

Während die Reaction Videos auf YouTube ohne Postproduktion auskommen, sind Reaction Videos, die außerhalb der Plattform verwendet werden, zumindest durch Schnitte verändert. Im Publikumsvideo des Neo Magazins stellt sich also die Frage nach der authentischen Darstellung unter anderem auch, weil die Beispiele nicht mit den einfachen, vermeintlich authentischen Mitteln der Amateuraufnahmen arbeiten. An diesen Beispielen tritt deutlich hervor, wie sehr die Rezeptionshaltung durch das Setting privater Räume und geringer Bildqualität die Rezepti- 
onshaltung vorgibt. In einer digitalen Bilderwelt, in der potenziell jedes Bild dem Manipulationsverdacht unterliegt - von den dargestellten Reaktionen gar nicht erst zu sprechen -, wird den Rezipient:innen etwas Authentisches präsentiert, das die Drastik der angeschauten Videos nochmals verstärkt. Vorwürfe, welche die Authentizität der Reaktionen anzweifeln, werden in den Kommentaren selten formuliert. Verlassen Reaction Videos diesen Kontext, müssen neue Rezeptionshaltungen gefunden werden; auch Fragen von Sichtbarkeitsverhältnissen und Authentizität stellen sich völlig neu.

Die Reaction Videos schaffen vor allem im Kontext von YouTube durch ihre Referenzialität die Grundlage für Kommunikation und Gemeinschaftsbildung. Im Spannungsfeld von Singularität und Diversität entstehen Gemeinschaften, die von Restriktion und moralischer Regulierung geprägt sind. Insbesondere in der Beobachtungsinstanz des invektiven Blickes zweiter Ordnung zeigt sich dieses regulative Potenzial. Diese Auseinandersetzung mit dem rezipierenden Subjekt, die imaginierte Beobachtungsinstanz des invektiven Blickes, ist eine wesentliche Produktionsbedingung des Reaction Videos und digitaler Bilder überhaupt. Insofern ist das Reaction Video Sinnbild von Digitalität. Denn der Produktion von Bildern begegnet es mit der Reproduktion und Modifikation immer neuer Bilder.

\section{Literaturverzeichnis}

Bataille, Georges (1981): Die Tränen des Eros [1961]. München: Matthes \& Seitz.

Butler, Judith (2005): Gefährdetes Leben. Politische Essays. Frankfurt a.M.: Suhrkamp. Franck, Georg (1998): Ökonomie der Aufmerksamkeit. Ein Entwurf. Wien: Hanser.

Freud, Sigmund (1958): Der Witz und seine Beziehungen zum Unbewussten [1905].

Frankfurt a.M.: S. Fischer.

Hentschel, Linda (2008): »Haupt oder Gesicht? Visuelle Gouvernementalität seit

9/11«. In: Dies. (Hg.): Bilderpolitik in Zeiten von Krieg und Terror. Medien, Macht und Geschlechterverhältnisse. Berlin: b-books, S. 183-202.

Klonk, Charlotte (2017): Terror - Wenn Bilder zu Waffen werden. Frankfurt a.M.: S. Fischer. DOI: 10.3790/hpb.66.1.14.

Kracauer, Siegfried (1964): Theorie des Films. Die Errettung der äußeren Wirklichkeit [1960]. In: Ders.: Siegfried Kracauer. Schriften. Bd. 3. Hg. v. Karsten Witte. Frankfurt a.M.: Suhrkamp.

Kristeva, Julia (1978): Die Revolution der poetischen Sprache [1974]. Frankfurt a.M.: Suhrkamp.

Kristeva, Julia (1982): Powers of Horror. An Essay on Abjection [1980]. New York: Columbia University Press.

Lacan, Jacques (1978): Die vier Grundbegriffe der Psychoanalyse [1964]. In: Ders.: Das Seminar von Jacques Lacan, Buch XI. Hg. v. Norbert Haas. Olten: Walter Verlag. 
Mau, Steffen (2018): Das metrische Wir. Über die Quantifizierung des Sozialen. Berlin: Suhrkamp.

Mead, George Herbert (1973): Geist, Identität und Gemeinschaft. Frankfurt a.M.: Suhrkamp.

Menninghaus, Winfried (2002): Ekel. Theorie und Geschichte einer starken Empfindung. Frankfurt a.M.: Suhrkamp.

Merleau-Ponty, Maurice (1986): Das Sichtbare und das Unsichtbare. Gefolgt von Arbeitsnotizen [1964]. München: Fink.

Meyer-Drawe, Käte (1990): Illusionen von Autonomie. München: P. Kirchheim Verlag. Otto, Isabell/Plohr, Nikola (2015): »Selfie-Technologie«. In: POP. Kultur und Kritik, Jg. 6, Nr. 1, S. 26-30. DOI: 10.14361/pop-2015-0104.

Reichert, Ramón (2015): »Selfie Culture. Kollektives Bildhandeln 2.0«. In: POP. Kultur und Kritik, Jg. 7, Nr. 2, S. 86-96. DOI: 10.14361/pop-2015-0214.

Sartre, Jean-Paul (1991): Das Sein und das Nichts. Versuch einer phänomenologischen Ontologie [1943]. In: Ders.: Sartre. Gesammelte Werke in Einzelausgaben. Philosophische Schriften. Bd. 3. Hg. v. Traugott König. Hamburg: Rowohlt.

Schachtner, Christina/Duller, Nicole (2014): »Kommunikationsort Internet. Digitale Praktiken und Subjektwerdung«. In: Carstensen, Tanja/Schachtner, Christina u.a. (Hgg.): Digitale Subjekte. Praktiken der Subjektivierung im Medienumbruch der Gegenwart. Bielefeld: transcript, S. 81-154. DOI: 10.14361/transcript.9783839422526.81.

Schankweiler, Kerstin (2019): Selfie-Proteste. Affektzeugenschaften und Bildökonomien in den Social Media. Berlin Working Paper SFB 1171 Affective Societies 05/16.

Schäfer, Fabian/Mühleder, Peter (2020): »Konnektiver Zynismus und Neue Rechte. Das Beispiel des YouTubers Adlersson«: in: Zeitschrift für Medienwissenschaft. Heft 22: Medium | Format, Jg. 12, Nr. 1, S. 130-149. DOI: 10.14361/zfmw-2020-120114.

Shifman, Limor (2011): "An anatomy of a YouTube meme«. In: new media \& society, Jg. 14, Nr. 2, S. 187-203. DOI: 10.1177/1461444811412160.

Shifman, Limor (2014): Meme. Kunst, Kultur und Politik im digitalen Zeitalter. Berlin: Suhrkamp.

Sontag, Susan (2003): Die Leiden anderer betrachten. München: Hanser.

Ullrich, Wolfgang (2019): Selfies. Berlin: Wagenbach. 


\section{Filmverzeichnis}

2 Girls 1 Cup Reaction - Robert Kelly (2007) (ErockFan): https://www.youtube.com /watch?v=QfvVN5DLT-c\&t=33s [letzter Zugriff 26.01.221].

2 Girls 1 Cup - Ü55 Reaction Vid-o - NEO MAGAZIN mit Jan Böhmermann in (2014) (ZDFneo): https://www.youTube.com/watch?v=DySkFqwaX14 [letzter Zugriff 26.01.2021].

2 girls and I cup reaction [daddy nd Ashley] =] (2009) (wtfitskelsey): https://www.y outube.com/watch?v=bShUwehhARg [letzter Zugriff 26.01.2021].

3 Guys 1 Hammer Reaction (2009) (wtfitskelsey): https://www.youtube.com/watch

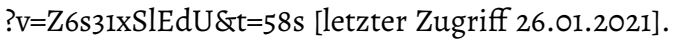

3 guys 1 hammer reaction (2012) (Kelcee Jane): eigenes Archivmaterial.

BEST Two Girls One Cup Reaction Video!!! LMAO!!! (2010) (Racardo Davis): https: //www.youtube.com/watch?v=_MsJgcOUs7o [letzter Zugriff 26.01.2021].

Emilys Video Reactions (2013) (Emilys Video): https://www.youtube.com/user/Em ilysVideoReactions [letzter Zugriff 26.01.2021].

Reacting To The Reaction Of The Reaction To The Reaction Of The Reaction Of The Reaction To The Reac (2017) (MrBeast): https://www.youtube.com/watch?v=rq UvnLPvbrM [letzter Zugriff 26.01.2021].

The Last da Vinci: The World is Watching (2017) (Christie's): https://www.youtube. com/watch?v=d7omwQLuGJQ [letzter Zugriff 26.01.2021].

\section{Abbildungsverzeichnis}

Abb. 1: Christie's: The Last da Vinci: The World is Watching (2017). Filmstill. https://ww w.youtube.com/watch? $=$ d7omwQLuGJQ [00:51].

Abb. 2: Ebd., [02:11].

Abb. 3: Ebd., [03:03].

Abb. 4: Jane, Kelcee: 3 guys I hammer reaction (2012), Filmstill des Reaction Videos aus eigenem Archivbestand, [00:42].

Abb. 5: Ebd., [01:36].

Abb. 6: Leng-Tsche, Folter der 100 Teile (1905). Aus: Bataille, Georges (1981): Die Tränen des Eros [1961]. München: Matthes \& Seitz, S. 245.

Abb. 7: wtfitskelsey: 2 girls and 1 cup reaction [daddy nd Ashley] =] (2009). Filmstill. htt ps://www.youtube.com/watch?v=bShUwehhARg [0:18].

Abb. 8: Ebd., [0:58].

Abb. 9: ErockFan: 2 Girls 1 Cup Reacti- $n$-Robert Kelly (2007). Filmstill. https://www. youtube.com/watch?v=QfvVN5DLT-c [0:28].

Abb. 10: Ebd., [01:14]. 
160 Johanna Heyne

Abb. 11: wtfitskelsey: 3 Guys I Hammer Reaction (2009). Filmstill. https://www.youTu be.com/watch?v=Z6s31xSlEdU\&t=1s [00:24].

Abb. 12: Ebd., [00:30].

Abb. 13: Racardo Davis: BEST Two Girls One Cup Reaction Video!!! LMAO!!! (2010). Filmstill. https://www.youtube.com/watch?v=_MsJgcOUs70 [01:58]. Abb. 14: Ebd., [02:16]. 